\title{
CONTINUOUS MIXTURES WITH BATHTUB-SHAPED FAILURE RATES
}

\author{
HENRY W. BLOCK, ${ }^{* * * * * *}$ University of Pittsburgh \\ YULIN LI, ${ }^{* * * *}$ The University of Toledo \\ THOMAS H. SAVITS **** AND \\ JIE WANG, ${ }^{*}$ University of Pittsburgh
}

\begin{abstract}
The failure rate of a mixture of even the most standard distributions used in reliability can have a complicated shape. However, failure rates of mixtures of two carefully selected distributions will have the well-known bathtub shape. Here we show that mixtures of whole families of distribtions can have a bathtub-shaped failure rate.
\end{abstract}

Keywords: Reliability; bathtub-shaped failure rate

2000 Mathematics Subject Classification: Primary 62N05

Secondary 90B25

\section{Introduction}

In this paper we consider continuous mixtures whose failure rate turns out to have a bathtub shape. For extensive discussions concerning mixtures of survival distributions and also bathtubshaped failure rate functions, see [16] and [17].

Most populations considered in a reliability setting can be considered to be heterogeneous. The reason for this is that there are usually at least two subpopulations: the normal (sometimes called the strong) subpopulation and the defective (or weak) subpopulation. This implies that the population can be modeled as a mixture of two or more lifetimes. The failure rate function for the population is not just a simple composite of the failure rate of the subpopulations. It is a complicated function whose shape is hard to predict, even if the shape of the failure rates of the subpopulations is very simple.

The history of such mixtures and their failure rates have long been studied in reliability. In a classic paper, Proschan [19] studied mixtures of exponential distributions and observed that the failure rate of the mixture was decreasing. It is intuitively obvious that the limit of the failure rate of the mixture of exponentials is the failure rate of the stongest exponential component, i.e. the one with the smallest failure rate. One of the first papers to state this result in the general case was Clarotti and Spizzichino [5]. Block et al. [3] gave a general version of this result and showed that, subject to mild technical assumptions, the asymptotic failure rate of a mixture is the limiting failure rate of the strongest subpopulation. A related result by Block and Joe [1] showed that, for a mixture of lifetimes, where the failure rate of the lifetimes are essentially

Received 2 November 2006; revision received 1 November 2007.

* Postal address: Department of Statistics, University of Pittsburgh, 2717 Cathedral of Learning, Pittsburgh, PA 15260, USA.

** Email address: hwb@stat.pitt.edu

*** Supported by NSA Grant H 98230-07-1-0018.

**** Postal address: Department of Mathematics, The University of Toledo, $2801 \mathrm{~W}$. Bancroft Street, Toledo, OH 43606-3390, USA. 
ratios of polynomials (as most well-known lifetime distributions are), the limiting distribution of the mixture possesses the same eventual monotonicity as the strongest component. For example, if the failure rate of the strongest component is eventually increasing, so is the failure rate of the mixture. A recent paper by Block et al. [2] gave improved versions of these two results. In this latter paper the initial behavior of mixtures was also discussed, as were the initial and eventual failure rates for systems of components.

Although, as described above, a lot is known about the asymptotic and initial behavior of the failure rate of mixtures, not a lot is known about the intermediate behavior. It has been observed that, for mixtures of lifetimes, the failure rate can be decreasing even at great age; see, e.g. [20]. The intermediate behavior of mixtures has been studied mostly for the mixture of two known distributions. See [12] and [13] for distributions mixed with exponentials, [4] for mixtures of increasing linear failure rates, [11] for mixtures of gamma distributions, [15] for mixtures of Weibulls, and [21] for a variety of mixtures of Weibulls and exponentials. Finkelstein, in a series of papers (see, e.g. [8]), has also discussed mixtures of failure rates in some special cases, such as frailty models.

In the present paper we go beyond mixtures of two distributions and consider the intermediate behavior for particular continuous mixtures of various distributions. In particular, we show how mixtures of whole families of distributions yield a distribution with a bathtub-shaped failure rate. Specifically, when a continuous mixture of exponentials, gammas, or Weibulls with decreasing failure rate are mixed with a gamma with increasing failure rate, a distribution with a bathtub-shaped failure rate is obtained. The form of the mixture we consider is

$$
f(t)=p h(t)+(1-p) g(t),
$$

where $h(t)$ is a continuous mixture of the type mentioned above, $0 \leq p \leq 1$, and $g(t)$ is the gamma distribution with increasing failure rate.

The basic result concerns the mixture of exponentials and is contained in Theorem 1 of Section 2. Section 3, Theorems 3 and 4, contains results about the mixture of gammas and the mixture of Weibulls. In Theorem 5 we give a result which describes some specific cases when mixtures of distributions with bathtub-shaped failure rates yield a distribution with a bathtub-shaped failure rate. Sections 4 and 5 contain some other special cases.

A few facts about the gamma distribution are needed. The density of the gamma with shape parameter $\alpha>0$ and scale parameter $\lambda>0$ is given by

$$
g(t \mid \alpha, \lambda)=\frac{\lambda^{\alpha}}{\Gamma(\alpha)} t^{\alpha-1} \mathrm{e}^{-\lambda t} \quad \text { for } t>0,
$$

and we use the notation $g(t \mid \alpha)$ when $\lambda=1$. We denote the survival functions by $\bar{G}(t \mid \alpha, \lambda)$ and by $\bar{G}(t \mid \alpha)$ for the $\lambda=1$ case. It is easy to check that

$$
\mathrm{e}^{t} \bar{G}(t \mid \alpha)=\frac{1}{\Gamma(\alpha)} \int_{0}^{\infty}(t+v)^{\alpha-1} \mathrm{e}^{-v} \mathrm{~d} v,
$$

and

$$
\frac{\mathrm{d}}{\mathrm{d} t}\left(\mathrm{e}^{t} g(t \mid \alpha)\right)=\mathrm{e}^{t} g(t \mid \alpha-1) \quad \text { and } \quad \frac{\mathrm{d}}{\mathrm{d} t}\left(\mathrm{e}^{t} \bar{G}(t \mid \alpha)\right)=\mathrm{e}^{t} \bar{G}(t \mid \alpha-1) .
$$

We now give the definition of a bathtub distribution.

Definition. A distribution has a bathtub-shaped failure rate if the failure rate function is decreasing up to a point and then increasing after that point. We sometimes use the term bathtub distribution to designate a distribution with bathtub-shaped failure rate function. 
Notes. 1. The terms decreasing and increasing will be used to mean nonincreasing and nondecreasing. If the monotonicity is strict, we will precede the terms with 'strictly'.

2. The distribution will be said to be degenerate bathtub if the failure rate function is always increasing or always decreasing. Otherwise it will be said to be nondegenerate bathtub.

\section{Main result}

It was recognized by several authors that distributions with bathtub-shaped failure rates could arise as simple mixtures. For example, Gupta and Warren [11, Section 3] showed that a certain mixture of two gammas, one with an increasing failure rate (IFR) and the other with a decreasing failure rate (DFR), could have a failure rate which is bathtub. An even simpler example, given by Block et al. [2, Example 2.4], gives a mixture of an exponential and an IFR gamma which also has a bathtub-shaped failure rate. It turns out that both of these examples are a special case of a much more general result. Mixing an IFR gamma with a host of different DFR distributions turns out to have a failure rate with bathtub shape. These results follow directly from Theorem 1, below, which shows that mixing an IFR gamma with a continuous mixture of exponentials yields a distribution with a bathtub-shaped failure rate.

Theorem 1. Consider a gamma distribution with density $g\left(t \mid \alpha, \lambda_{0}\right)$, with $\alpha>2$ and $\lambda_{0}>0$, and a family of exponentials with parameters $\lambda>\lambda_{0}$. Let $\mathrm{P}$ be a probability measure whose support set $S$ is a subset of $\left(\lambda_{0}, \infty\right)$. The resulting mixture, with density

$$
f(t)=p \int \lambda \mathrm{e}^{-\lambda t} \mathrm{P}(\mathrm{d} \lambda)+q g\left(t \mid \alpha, \lambda_{0}\right)
$$

where $p+q=1, p>0, q>0$, has a bathtub-shaped failure rate. If $\mathrm{P}$ has a finite first moment then the failure rate is nondegenerate bathtub.

Remark. The assumption on $\mathrm{P}$ that its support set $S$ is a subset of $\left(\lambda_{0}, \infty\right)$ implies that $\inf \{x: \mathrm{P}((-\infty, x])>0\}>\lambda_{0}$.

Proof of Theorem 1. It is enough to prove the theorem for $\lambda_{0}=1$ and then to rescale. The proof can be carried out for most parameter choices by directly examining the failure rate $r(t)=f(t) / \bar{F}(t)$, where $\bar{F}(t)$ is the survival function. However, the proof is easiest and most complete using a result of [9], which examined the function $\eta(t)=-f^{\prime}(t) / f(t)$ for $t>0$. This result states that if $\eta(t)$ is bathtub-shaped then the failure rate is either increasing or has a nondegenerate bathtub shape.

Using the gamma notation of Section 2, we find that

$$
\eta(t)=\frac{p \int \lambda^{2} \exp (-(\lambda-1) t) \mathrm{P}(\mathrm{d} \lambda)+q \mathrm{e}^{t} g(t \mid \alpha)-q \mathrm{e}^{t} g(t \mid \alpha-1)}{p \int \lambda \exp (-(\lambda-1) t) \mathrm{P}(\mathrm{d} \lambda)+q \mathrm{e}^{t} g(t \mid \alpha)},
$$

and so

$$
\eta^{\prime}(t)=\frac{q^{2} A(t)+p^{2} B(t)+p q C(t)}{\left(p \int \lambda \exp (-(\lambda-1) t) \mathrm{P}(\mathrm{d} \lambda)+q \mathrm{e}^{t} g(t \mid \alpha)\right)^{2}} .
$$


Here

$$
\begin{aligned}
A(t)= & \mathrm{e}^{t}(g(t \mid \alpha-1)-g(t \mid \alpha-2)) \mathrm{e}^{t} g(t \mid \alpha) \\
& -\mathrm{e}^{t}(g(t \mid \alpha)-g(t \mid \alpha-1)) \mathrm{e}^{t} g(t \mid \alpha-1) \\
= & \frac{\alpha-1}{(\Gamma(\alpha))^{2}} t^{2(\alpha-2)}, \\
B(t)= & \int \lambda^{2} \exp (-(\lambda-1) t) \mathrm{P}(\mathrm{d} \lambda) \int \xi(\xi-1) \exp (-(\xi-1) t) \mathrm{P}(\mathrm{d} \xi) \\
& -\int \lambda^{2}(\lambda-1) \exp (-(\lambda-1) t) \mathrm{P}(\mathrm{d} \lambda) \int \xi \exp (-(\xi-1) t) \mathrm{P}(\mathrm{d} \xi) \\
= & -\int\left(\int_{[\xi, \infty)} \lambda \xi(\lambda-\xi)^{2} \exp (-(\lambda+\xi-2) t) \mathrm{P}(\mathrm{d} \lambda)\right) \mathrm{P}(\mathrm{d} \xi),
\end{aligned}
$$

and

$$
\begin{aligned}
C(t)= & -\int \lambda^{2}(\lambda-1) \exp (-(\lambda-1) t) \mathrm{P}(\mathrm{d} \lambda) \mathrm{e}^{t} g(t \mid \alpha) \\
& +\int \lambda \exp (-(\lambda-1) t) \mathrm{P}(\mathrm{d} \lambda)\left(\mathrm{e}^{t} g(t \mid \alpha-1)-\mathrm{e}^{t} g(t \mid \alpha-2)\right) \\
& -\int \lambda^{2} \exp (-(\lambda-1) t) \mathrm{P}(\mathrm{d} \lambda) \mathrm{e}^{t} g(t \mid \alpha-1) \\
& +\int \lambda(\lambda-1) \exp (-(\lambda-1) t) \mathrm{P}(\mathrm{d} \lambda)\left(\mathrm{e}^{t} g(t \mid \alpha)-\mathrm{e}^{t} g(t \mid \alpha-1)\right) \\
= & -\int \lambda(\lambda-1)^{2} \exp (-(\lambda-1) t) \mathrm{P}(\mathrm{d} \lambda) \mathrm{e}^{t} g(t \mid \alpha) \\
& -2 \int \lambda(\lambda-1) \exp (-(\lambda-1) t) \mathrm{P}(\mathrm{d} \lambda) \mathrm{e}^{t} g(t \mid \alpha-1) \\
& -\int \lambda \exp (-(\lambda-1) t) \mathrm{P}(\mathrm{d} \lambda) \mathrm{e}^{t} g(t \mid \alpha-2) .
\end{aligned}
$$

Note that, for all $t>0, A(t) \geq 0, B(t) \leq 0$, and $C(t) \leq 0$.

To show that $\eta$ is bathtub shaped, we need only show that the function

$$
h(t)=q^{2} A(t)+p^{2} B(t)+p q C(t)
$$

has only one sign change and it is from negative to positive. We first consider the sign of $h(0+)=q^{2} A(0+)+p^{2} B(0+)+p q C(0+)$. For $\alpha>2, A(0+)=0$, and so $h(0+) \leq 0$. If $\mathrm{P}$ is nondegenerate then

$$
h(0+) \leq-p^{2} B(0+)=-p^{2} \int\left(\int_{(\xi, \infty)} \lambda \xi(\lambda-\xi)^{2} \mathrm{P}(\mathrm{d} \lambda)\right) \mathrm{P}(\mathrm{d} \xi)<0 .
$$

If $\mathrm{P}$ is degenerate at $\lambda_{1}>\lambda_{0}=1$ then $B(t)=0$ and so $h(t)$ behaves like $q^{2} A(t)+p q C(t)$ as $t \downarrow 0$, where

$$
\begin{aligned}
\Gamma(\alpha) C(t)=- & \exp \left(-\left(\lambda_{1}-1\right) t\right) \\
& \times\left(\lambda_{1}\left(\lambda_{1}-1\right)^{2} t^{\alpha-1}+2(\alpha-1) \lambda_{1}\left(\lambda_{1}-1\right) t^{\alpha-2}+(\alpha-1)(\alpha-2) \lambda_{1} t^{\alpha-3}\right) .
\end{aligned}
$$


Clearly, for $2<\alpha<3, C(0+)=-\infty$; for $\alpha=3, C(0+)=-(\alpha-1)(\alpha-2) \lambda_{1} / 2$. Thus, we conclude that $h(0+)<0$ when $2<\alpha \leq 3$. For $\alpha>3$, however, $h(0+)=0$ since $C(0+)=0$. In this case we can rewrite $h(t)$ as

$$
\begin{gathered}
h(t)=t^{\alpha-3}\left(q^{2} \frac{\alpha-1}{(\Gamma(\alpha))^{2}} t^{\alpha-1}-p q \frac{\exp \left(-\left(\lambda_{1}-1\right) t\right)}{\Gamma(\alpha)}\left(\lambda_{1}\left(\lambda_{1}-1\right)^{2} t^{2}+2(\alpha-1) \lambda_{1}\left(\lambda_{1}-1\right) t\right.\right. \\
\left.\left.+\lambda_{1}(\alpha-1)(\alpha-2)\right)\right) .
\end{gathered}
$$

Consequently, $h(t) / t^{\alpha-3} \rightarrow-p q(\alpha-1)(\alpha-2) \lambda_{1} / \Gamma(\alpha)$ as $t \downarrow 0$, and so $h(t)<0$ in a neighborhood of $t=0+$. Thus, we conclude that $h(t)<0$ for small values of $t>0$ for all $\alpha>2$.

Also, since the support $S$ of $\mathrm{P}$ is by definition a closed set, it does not contain a neighborhood of 1 and so it follows that $B(t) \rightarrow 0$ and $C(t) \rightarrow 0$ as $t \rightarrow \infty$. Since $A(t) \rightarrow \infty$ as $t \rightarrow \infty$ for $\alpha>2$, we conclude that $h(t)>0$ for large $t$ and, consequently, there exists at least one positive root of $h(t)=0$. To show that there exists only one such root, it suffices to show that if $h\left(t_{0}\right)=0$ for $0<t_{0}<\infty$ then $h^{\prime}\left(t_{0}\right)>0$. So, let

$$
0=h\left(t_{0}\right)=q^{2} A\left(t_{0}\right)+p^{2} B\left(t_{0}\right)+p q C\left(t_{0}\right),
$$

and consider

$$
h^{\prime}\left(t_{0}\right)=q^{2} A^{\prime}\left(t_{0}\right)+p^{2} B^{\prime}\left(t_{0}\right)+p q C^{\prime}\left(t_{0}\right)
$$

Using the facts that

$$
q^{2} A^{\prime}\left(t_{0}\right)=q^{2} \frac{2(\alpha-1)(\alpha-2)}{\Gamma^{2}(\alpha)} t_{0}^{2 \alpha-5}=q^{2} \frac{2(\alpha-2)}{t_{0}} A\left(t_{0}\right)
$$

and $q^{2} A\left(t_{0}\right)=-p^{2} B\left(t_{0}\right)-p q C\left(t_{0}\right)$, it follows, after some simplification, that

$$
\begin{aligned}
h^{\prime}\left(t_{0}\right)= & \frac{2(\alpha-2)}{t_{0}}\left(-p^{2} B\left(t_{0}\right)\right) \\
& +p^{2} \int\left(\int_{[\xi, \infty)} \lambda \xi(\lambda-\xi)^{2}(\lambda+\xi-2) \exp \left(-(\lambda+\xi-2) t_{0}\right) \mathrm{P}(\mathrm{d} \lambda)\right) \mathrm{P}(\mathrm{d} \xi) \\
& +\frac{p q}{\Gamma(\alpha)}\left(t_{0}^{\alpha-1} \int \lambda(\lambda-1)^{3} \exp \left(-(\lambda-1) t_{0}\right) \mathrm{P}(\mathrm{d} \lambda)\right. \\
& +(3 \alpha-5) t_{0}^{\alpha-2} \int \lambda(\lambda-1)^{2} \exp \left(-(\lambda-1) t_{0}\right) \mathrm{P}(\mathrm{d} \lambda) \\
& +3(\alpha-1)(\alpha-2) t_{0}^{\alpha-3} \int \lambda(\lambda-1) \exp \left(-(\lambda-1) t_{0}\right) \mathrm{P}(\mathrm{d} \lambda) \\
& \left.+(\alpha-1)^{2}(\alpha-2) t_{0}^{\alpha-4} \int \lambda \exp \left(-(\lambda-1) t_{0}\right) \mathrm{P}(\mathrm{d} \lambda)\right)
\end{aligned}
$$

Hence, $h^{\prime}\left(t_{0}\right)>0$.

As noted previously, we can thus conclude that $h(t)<0$ for $0<t<t_{0}$ and $h(t)>0$ for $t>t_{0}$. This implies that $\eta(t)$ is bathtub shaped and the result follows from [9]. Now suppose that $\mathrm{P}$ has a finite first moment. Then, from Remark 2.4 of [2], we deduce that

$$
r^{\prime}(0+)=-p \int \lambda^{2} \mathrm{P}(\mathrm{d} \lambda)+p^{2}\left(\int \lambda \mathrm{P}(\mathrm{d} \lambda)\right)^{2}<0 .
$$

Hence, $r(t)$ must be nondegenerate bathtub. 
Remarks. (i) The results of [2] allow us (under certain conditions) to conclude that the failure rate of the mixture, (1), initially decreases and then ultimately increases to $\lambda_{0}$; however, this is not enough to conclude that $r(t)$ has a bathtub shape.

(ii) We can also show that the failure rate is nondegenerate bathtub if $\mathrm{P}$ is nondegenerate with support which is a subset of $\left(2 \lambda_{0}, \infty\right)$.

\section{Applications of Theorem 1}

In Section 2 we considered mixtures of the form

$$
f(t)=p h(t)+q g(t),
$$

where $g(t)=g\left(t \mid \alpha, \lambda_{0}\right)$ was the density of a gamma distribution with shape parameter $\alpha>2$ and scale parameter $\lambda_{0}>0$, and $h(t)=\int \lambda \mathrm{e}^{-\lambda t} \mathrm{P}(\mathrm{d} \lambda)$ was a continuous mixture of exponential densities with $\mathrm{P}$ being a probability distribution having support which is a subset of $\left(\lambda_{0}, \infty\right)$.

We note that the survival function $\bar{H}(t)$ of $h(t)$ is given by

$$
\bar{H}(t)=\int \mathrm{e}^{-\lambda t} \mathrm{P}(\mathrm{d} \lambda) .
$$

According to Theorem 1 of [7], it follows that $\bar{H}(t)$ must be a completely monotone function (i.e. $(-1)^{n} \mathrm{~d}^{n} \bar{H}(t) / \mathrm{d} t^{n} \geq 0$ for $t>0$ ). Conversely, if a survival function $\bar{H}(t)$ is completely monotone then there exists a unique probability measure $\mathrm{P}$ on $[0, \infty)$ such that $(2)$ holds. Thus, we can reformulate Theorem 1 as follows.

Theorem 2. (Theorem 1 reformulated.) Consider the survival function $\bar{F}(t)$ of the form

$$
\bar{F}(t)=p \bar{H}(t)+q \bar{G}\left(t \mid \alpha, \lambda_{0}\right),
$$

where $p+q=1, p>0, q>0, \lambda_{0}>0, \alpha>2$, and $\bar{H}(t)$ is a completely monotone function, whose associated probability measure $\mathrm{P}$ in (2) has support which is a subset of $\left(\lambda_{0}, \infty\right)$. Then $\bar{F}(t)$ has a bathtub-shaped failure rate. If, in addition, $-\bar{H}^{\prime}(0+)=\int \lambda \mathrm{P}(\mathrm{d} \lambda)<\infty$ then it is nondegenerate bathtub.

Our main results in this section are applications of Theorem 2 and the following simple lemma.

Lemma 1. Let $(\Theta, Q)$ be a probability space, and let $\left\{\phi_{\theta}: \theta \in \Theta\right\}$ be a family of completely monotone functions jointly measurable in $(\theta, t) \in \Theta \times[0, \infty)$. Then

$$
\phi(t)=\int_{\Theta} \phi_{\theta}(t) \mathrm{Q}(\mathrm{d} \theta)
$$

is completely monotone.

Proof. By (2) we know that, for every $\theta \in \Theta$, there exists a unique probability measure $\mathrm{P}_{\theta}$ on $[0, \infty)$ such that

$$
\phi_{\theta}(t)=\int_{[0, \infty)} \mathrm{e}^{-\lambda t} \mathrm{P}_{\theta}(\mathrm{d} \lambda)
$$

Hence,

$$
\phi(t)=\int_{\Theta}\left(\int_{[0, \infty)} \mathrm{e}^{-\lambda t} \mathrm{P}_{\theta}(\mathrm{d} \lambda)\right) \mathrm{Q}(\mathrm{d} \theta)=\int_{[0, \infty)} \mathrm{e}^{-\lambda t} \mathrm{R}(\mathrm{d} \lambda),
$$


where $\mathrm{R}$ is a probability measure on $[0, \infty)$ given by

$$
\mathrm{R}(A)=\int_{\Theta} \mathrm{P}_{\theta}(A) \mathrm{Q}(\mathrm{d} \theta)
$$

for all Borel subsets $A$ of $[0, \infty)$ (see [18, Theorem T16]). Thus, (3) shows that $\phi$ is completely monotone.

Our results in this section involve specific mixtures which give rise to bathtub distributions. These results are of the following types:

1. mixtures of DFR gamma distributions with an IFR gamma distribution are bathtub,

2. mixtures of DFR 'Weibull' distributions with an IFR gamma are bathtub,

3. mixtures of certain bathtub distributions are bathtub.

The above types are quite remarkable since recent research has shown that mixtures of distributions with even the simplest failure rate functions can lead to vastly different monotonic behavior. For example, in [4] it was shown that the mixture of two distributions with increasing linear failure rates can have four changes of monotonicity. Moreover, results concerning the behavior of mixtures of more than two distributions are very sparse in the literature, while the above results involve possibly continuous mixtures.

We first show that arbitrary mixtures of DFR gamma distributions with an IFR gamma distribution have a bathtub-shaped failure rate.

Theorem 3. Consider an arbitrary mixture of DFR gamma densities $g(t \mid \beta, \xi)$ with $0<$ $\beta \leq 1$ and $\xi>0$, and let $\lambda_{0}>0$. Let $\mathrm{Q}$ be a probability measure on $\mathbb{R}^{2}$ with support which is a subset of $(0,1] \times\left(\lambda_{0}, \infty\right)$. Then the mixture distribution

$$
f(t)=p \int_{\mathbb{R}^{2}} g(t \mid \beta, \xi) \mathrm{dQ}(\beta, \xi)+q g\left(t \mid \alpha, \lambda_{0}\right),
$$

where $p+q=1, p>0, q>0$, has a bathtub-shaped failure rate.

Proof. According to [10], any DFR gamma is a mixture of exponentials, i.e. if $0<\beta \leq 1$ and $\xi>0$ then the survival function can be written as

$$
\bar{G}(t \mid \beta, \xi)=\int_{[0, \infty)} \mathrm{e}^{-\lambda t} \mathrm{P}_{(\beta, \xi)}(\mathrm{d} \lambda),
$$

where $\mathrm{P}_{(\beta, \xi)}$ is a probability measure on $[0, \infty)$ having density

$$
p_{(\beta, \xi)}(\lambda)=\frac{(\lambda-\xi)^{-\beta} \xi^{\beta}}{\lambda \Gamma(1-\beta) \Gamma(\beta)} \mathbf{1}_{(\xi, \infty)}(\lambda)
$$

But, from Lemma 1, it follows that we can write

$$
\bar{G}(t)=\int_{\mathbb{R}^{2}} \bar{G}(t \mid \beta, \xi) \mathrm{dQ}(\beta, \xi)=\int_{[0, \infty)} \mathrm{e}^{-\lambda t} \mathrm{dR}(\lambda),
$$

where $\mathrm{R}(A)=\int_{\mathbb{R}^{2}} \mathrm{P}_{(\beta, \xi)}(A) \mathrm{dQ}(\beta, \xi)$ for all Borel subsets $A \subset[0, \infty)$. Using our assumptions on $\mathrm{Q}$, it is not hard to show that $\mathrm{R}$ has support which is a subset of $\left(\lambda_{0}, \infty\right)$. The result then follows from Theorem 2. 
Remark. Since the probability measure R above does not have a finite first moment, we cannot use Theorem 2 to conclude that the failure rate is nondegenerate bathtub. However, if the support of $\mathrm{Q}$ is a subset of $(0,1] \times\left(2 \lambda_{0}, \infty\right)$, it follows that the support of $\mathrm{R}$ is a subset of $\left(2 \lambda_{0}, \infty\right)$. Since $\mathrm{R}$ is clearly nondegenerate, we conclude from the remark following Theorem 1 that the failure rate of the mixture is nondegenerate bathtub.

Our next result is similar to Theorem 3, but with DFR 'Weibull' distributions. The 'Weibull' distributions we consider are a variant of the usual Weibull distributions since the failure rate of the usual nondegenerate (i.e. not an exponential) DFR Weibull distribution decreases to 0 . The variant we consider has the survival funtion

$$
\bar{H}(t \mid \beta, c, \xi)=\mathrm{e}^{-\xi t} \bar{W}(t \mid \beta, c), \quad \xi>0,
$$

where $\xi>0$ and $\bar{W}(t \mid \beta, c)$ is the survival function of the usual DFR Weibull distribution with scale parameter $c>0$ and shape parameter $\beta$ with $0<\beta<1$, i.e.

$$
\bar{W}(t \mid \beta, c)=\exp \left(-(c t)^{\beta}\right), \quad t>0 .
$$

For this variant of the Weibull distribution, the failure rate decreases to $\xi$ as $t$ tends to $\infty$. We call this a 'Weibull' distribution with parameters $(\beta, c, \xi)$.

Theorem 4. Consider any family of DFR 'Weibull' distributions of the form of (4), and let $\lambda_{0}>0$. Let $\mathrm{Q}$ be a probability measure on $\mathbb{R}^{3}$ with support which is a subset of $(0,1] \times$ $(0, \infty) \times\left(\lambda_{0}, \infty\right)$. Then the mixture with survival function

$$
\bar{F}(t)=p \int_{\mathbb{R}^{3}} \bar{H}(t \mid \beta, c, \xi) \mathrm{dQ}(\beta, c, \xi)+q \bar{G}\left(t \mid \alpha, \lambda_{0}\right)
$$

has a bathtub-shaped failure rate for $\alpha>2$.

Proof. Jewell [14] claimed that any DFR Weibull distribution is a mixture of exponentials. Thus, for $\bar{W}$ given in (5), we can write

$$
\bar{W}(t \mid \beta, c)=\int_{[0, \infty)} \mathrm{e}^{-\lambda t} \mathrm{P}_{(\beta, c)}(\mathrm{d} \lambda)
$$

for some probability measure $\mathrm{P}_{(\beta, c)}$ on $[0, \infty)$. Hence,

$$
\bar{H}(t \mid \beta, c, \xi)=\int_{[0, \infty)} \exp (-(\lambda+\xi) t) \mathrm{P}_{(\beta, c)}(\mathrm{d} \lambda)=\int \mathrm{e}^{-\gamma t} \mathrm{P}_{(\beta, c, \xi)}(\mathrm{d} \gamma)
$$

where $\mathrm{P}_{(\beta, c, \xi)}$ is the shifted version of $\mathrm{P}_{(\beta, c)}$, i.e. for every bounded Borel function $\psi$ on $[0, \infty)$,

$$
\int_{[0, \infty)} \psi(\gamma) \mathrm{P}_{(\beta, c, \xi)}(\mathrm{d} \gamma)=\int_{[0, \infty)} \psi(\lambda+\xi) \mathrm{P}_{(\beta, c)}(\mathrm{d} \lambda) .
$$

Note that the support of $\mathrm{P}_{(\beta, c, \xi)}$ is contained in $[\xi, \infty)$. By Lemma 1 we can write

$$
\bar{H}(t)=\int_{\mathbb{R}^{3}} \bar{H}(t \mid \beta, c, \xi) \mathrm{dQ}(\beta, c, \xi)=\int_{[0, \infty)} \mathrm{e}^{-\gamma t} \mathrm{R}(\mathrm{d} \gamma),
$$

with

$$
\mathrm{R}(A)=\int_{\mathbb{R}^{3}} \mathrm{P}_{(\beta, c, \xi)}(A) \mathrm{dQ}(\beta, c, \xi) .
$$

Hence, again the result follows from Theorem 2 since $\mathrm{R}$ has support which is a subset of $\left(\lambda_{0}, \infty\right)$. 
Remark. As noted in the proof, Jewell [14] claimed, without proof, that a DFR Weibull survival function is completely monotone. The easiest proof that we know is a nice application of Faà di Bruno's formulae (see [6]) and we include it here. It suffices to consider the case in which $c=1$ and $0<\beta \leq 1$, and let $\phi(t)=\exp \left(-t^{\beta}\right), t>0$. We can write $\phi$ as a composition $\phi(t)=a(b(t))$, where $a(x)=\mathrm{e}^{-x}$ and $b(t)=t^{\beta}$. According to Faà di Bruno's formulae

$$
\frac{\mathrm{d}^{n}}{\mathrm{~d} t^{n}} \phi(t)=\sum_{k=1}^{n} \frac{\mathrm{d}^{k}}{\mathrm{~d} x^{k}} a(b(t)) \sum_{p(n, k)} n ! \prod_{i=1}^{n} \frac{\left(\mathrm{d}^{i} b(t) / \mathrm{d} t^{i}\right)^{\lambda_{i}}}{\lambda_{i} !(i !)^{\lambda_{i}}}
$$

where $p(n, k)=\left\{\left(\lambda_{1}, \ldots, \lambda_{n}\right): \lambda_{i} \geq 0, \sum_{i=1}^{n} \lambda_{i}=k, \sum_{i=1}^{n} i \lambda_{i}=n\right\}$. Since

$$
\frac{\mathrm{d}^{k}}{\mathrm{~d} x^{k}}(a(b(t)))=(-1)^{k} \phi(t)
$$

to show that $(-1)^{n} \mathrm{~d}^{n} \phi(t) / \mathrm{d} t^{n} \geq 0$ for $n \geq 1$ and $t>0$, it suffices to show that each term

$$
(-1)^{n+k} \prod_{i=1}^{n} \frac{\left(\mathrm{d}^{i} b(t) / \mathrm{d} t^{i}\right)^{\lambda_{i}}}{\lambda_{i} !(i !)^{\lambda_{i}}} \geq 0
$$

But,

$$
(-1)^{i+1} \frac{\mathrm{d}^{i}}{\mathrm{~d} t^{i}} b(t)=(-1)^{i+1} \beta(\beta-1) \cdots(\beta-i+1) t^{\beta-i} \geq 0, \quad 0<\beta \leq 1 .
$$

Since $\left(\lambda_{1}, \ldots, \lambda_{n}\right) \in p(n, k)$

$$
\sum_{i=1}^{n}(i+1) \lambda_{i}=n+k
$$

and we are done.

Finally, we have a closure result for any bathtub distributions which arise in any of the above manners (i.e. from Theorems 1, 3, or 4).

Theorem 5. The mixture of any bathtub distributions which arise as a mixture of exponentials, DFR gammas, or DFR Weibulls with the same IFR gamma having shape parameter $\alpha>2$ has a bathtub-shaped distribution.

Proof. Since this will be a mixture of exponentials with an IFR gamma having shape parameter $\alpha>2$, it will have a bathtub shape.

\section{The $\alpha=2$ case}

The results for the special case in which $\alpha=2$ are similar to Theorem 1 , but a slightly different proof is required.

Theorem 6. Consider a gamma distribution with density $g\left(t \mid \alpha, \lambda_{0}\right)$, where $\alpha=2$ and $\lambda_{0}>0$, and a family of exponentials with parameters $\lambda>\lambda_{0}$. Let $\mathrm{P}$ be a probability measure whose support set $S$ is a subset of $\left(\lambda_{0}, \infty\right)$. The resulting mixture, with density

$$
f(t)=p \int \lambda \mathrm{e}^{-\lambda t} \mathrm{P}(\mathrm{d} \lambda)+q g\left(t \mid 2, \lambda_{0}\right),
$$


where $p+q=1, p>0, q>0$, has a bathtub-shaped failure rate $r(t)$. If $\mathrm{P}$ has a finite first moment then the failure rate is nondegenerate bathtub if

$$
p \int \lambda^{2} \mathrm{P}(\mathrm{d} \lambda)-p^{2}\left(\int \lambda \mathrm{P}(\mathrm{d} \lambda)\right)^{2}>q
$$

otherwise, it is increasing.

Proof. Without loss of generality, we need consider only the case in which $\lambda_{0}=1$. As in the proof of Theorem 1, we show that $\eta(t)$ is bathtub, or equivalently, that $h(t)$ is bathtub. In this case, $h(t)=q^{2} A(t)+p^{2} B(t)+p q C(t)$, where

$$
\begin{aligned}
A(t) & =1 \\
B(t) & =-\int\left(\int_{[\xi, \infty)} \lambda \xi(\lambda-\xi)^{2} \exp (-(\lambda+\xi-2) t) \mathrm{P}(\mathrm{d} \lambda)\right) \mathrm{P}(\mathrm{d} \xi), \\
C(t) & =-\left(t \int \lambda(\lambda-1)^{2} \exp (-(\lambda-1) t) \mathrm{P}(\mathrm{d} \lambda)+2 \int \lambda(\lambda-1) \exp (-(\lambda-1) t) \mathrm{P}(\mathrm{d} \lambda)\right) .
\end{aligned}
$$

It is easy to see that $h(t) \rightarrow q^{2}$ as $t$ increases to $\infty$, and thus $h(t)>0$ for large $t$. Also, as in the proof of Theorem 1 , we can show that if $h\left(t_{0}\right)=0$ for some $0<t_{0}<\infty$ then $h^{\prime}\left(t_{0}\right)>0$. Consequently, $h(t)$ can have at most one 0. Moreover, if it has a 0 , the sign of $h(t)$ goes from negative to positive. We thus conclude that $\eta(t)$ is bathtub. From [9], it follows that the failure rate is either increasing or nondegenerate bathtub.

Now suppose that $\mathrm{P}$ has a finite first moment. In this case we can examine the value of $r^{\prime}(0+)$ :

$$
r^{\prime}(0+)=-p \int \lambda^{2} \mathrm{P}(\mathrm{d} \lambda)+p^{2}\left(\int \lambda \mathrm{P}(\mathrm{d} \lambda)\right)^{2}+q .
$$

Thus, the failure rate is increasing if $r^{\prime}(0+) \geq 0$, while it is nondegenerate bathtub if $r^{\prime}(0+)<0$.

\section{Other cases}

In Section 2 we examined the mixture (1) for the case of a mixture of exponentials and an IFR gamma distribution with shape parameter $\alpha>2$. The following questions arise immediately.

1. What happens when the mixture (1) consists of a mixture of exponentials and an IFR gamma with $1<\alpha<2$ ?

2. Why not consider a mixture of exponentials with a mixture of IFR gammas?

The second question is easy to answer since mixtures of IFR gammas are not generally IFR. Gupta and Warren [11] gave an example of a mixture of two IFR gammas where the failure rate had two changes of monotonicity. Mixing this with a mixture of exponentials would most certainly give a distribution which does not have a bathtub shape.

The first question is unsolved except in special cases. See [11] for cases where a mixture of this type does not have a bathtub shape.

\section{Acknowledgement}

We thank an anonymous referee for some useful comments. 


\section{References}

[1] Block, H. And Joe, H. (1997). Tail behavior of the failure rate functions of mixtures. Lifetime Data Anal. 3, 269-288.

[2] Block, H. W., LI, Y. AND SAVITs, T. H. (2003). Initial and final behavior of failure rate functions for mixtures and systems. J. Appl. Prob. 40, 721-740.

[3] Block, H. W., Mi, J. and Savits, T. H. (1993). Burn-in and mixed populations. J. Appl. Prob. 30, $692-702$.

[4] Block, H. W., Savits, T. H. and Wondmagegnehu, E. (2003). Mixtures of distributions with linear failure rates. J. Appl. Prob. 40, 485-504.

[5] Clarotti C. A. and Spizzichino, F. (1990). Bayes burn-in decision procedure. Prob. Eng. Inf. Sci. 4, 437-445.

[6] Constantine, G. M. and Savits, T. H. (1996). A multivariate Faà di Bruno formula with applications. Trans. Amer. Math. Soc. 348, 503-520.

[7] Feller, W. (1966). An Introduction to Probability Theory and Its Applications, Vol II. John Wiley, New York.

[8] Finkelstein, M. S. and Esaulova, V. (2001). Why the mixture failure rate decreases. Reliab. Eng. System Safety 71, 173-177.

[9] Glaser, R. E. (1980). Bathtub and related failure rate characterizations. J. Amer. Statist. Assoc. 75, 667-672.

[10] Gleser, L. J. (1989). The gamma distribution as a mixture of exponential distributions. Amer. Statist. 43, 115-117.

[11] Gupta, R. C. AND Warren, R. (2001). Determination of change points of non-monotonic failure rates. Commun. Statist. Theory Meth. 30, 1903-1920.

[12] Gurland, J. and Sethuraman, J. (1994). Reversal of increasing failure rates when pooling failure data. Technometrics 36, 416-418.

[13] Gurland, J. and Sethuraman, J. (1995). How pooling failure data may reverse increasing failure rates. J. Amer. Statist. Assoc. 90, 1416-1423.

[14] Jewell, N. P. (1982). Mixtures of exponential distributions. Ann. Statist. 10, 479-484.

[15] Jiang, R. And Murthy, D. N. P. (1998). Mixture of Weibull distributions-parametric characterization of failure rate functions. Appl. Stoch. Models Data Anal. 14, 47-65.

[16] LaI, C.-D. AND XIE, M. (2006). Stochastic Ageing and Dependence for Reliability. Springer, New York.

[17] Marshall, A. W. And Olkin, I. (2007). Life Distributions. Springer, New York.

[18] Meyer, P. A. (1966). Probability and Potentials. Blaisdell, Waltham, MA.

[19] Proschan, F. (1963). Theoretical explanation of observed decreasing failure rate. Technometrics 5, 373-383.

[20] Wang, J.-L., Müller, H. And CAPra, W. B. (1998). Analysis of oldest-old mortality: lifetables revisited. Ann. Statist. 26, 126-163.

[21] Wondmagegnehu, E. T., Navarro, J. and Hernandez, P. J. (2005). Bathtub shaped failure rate from mixtures: a practical point of view. IEEE Trans. Reliab. 54, 270-275. 